\title{
HESITANT FUZZY SETS APPROACH TO IDEAL THEORY IN TERNARY SEMIGROUPS
}

\author{
A. Fairooze Talee ${ }^{1 \S}$, M. Yahya Abbasi ${ }^{2}$, S. Ali Khan ${ }^{3}$ \\ ${ }^{1,2,3}$ Department of Mathematics \\ Jamia Millia Islamia \\ New Delhi - 110 025, INDIA
}

\begin{abstract}
In this paper, we introduce hesitant fuzzy ideals on ternary semigroups and define hesitant fuzzy left (resp. lateral, right)ideals on ternary semigroups. Here we define hesitant fuzzy point on ternary semigroups and prove some interesting results on them. We also characterize regular ternary semigroups in terms of hesitant fuzzy left, hesitant fuzzy lateral and hesitant fuzzy right ideals using hesitant fuzzy points.
\end{abstract}

AMS Subject Classification: 20M12, 20N99, 08A72

Key Words: ternary semigroups, hesitant fuzzy ideals

\section{Introduction and Preliminaries}

The idea of investigation of $n$-ary algebras, i.e. the sets with one $n$-ary operation was given by Kasner [4]. A ternary semigroup is a particular case of an $n$-ary semigroup for $n=3$, [5]. The ternary semigroups are universal algebras with one associative operation. The ideal theory in ternary semigroup was studied by Sioson [7].

Zadeh [11] introduced the concept of fuzzy set as an extension of the classical notion of set. The fuzzy set theory can be used in a wide range of domains to manipulate different types of uncertainties. Torra $[8,9]$ introduced hesitant

$\begin{array}{ll}\text { Received: January 20, } 2018 & \text { (c) } 2018 \text { Academic Publications }\end{array}$

$\S$ Correspondence author 
fuzzy sets (briefly HFSs) as a new generalization of fuzzy set. Hesitant fuzzy set is used to control hesitant situation which were difficult to handle by previous extensions. Jun et. al. [2] applied the HFSs in semigroups. Abbasi et al. [6] applied the notion of HFSs to ordered semigroups.

Kar and Sarkar [3] introduced fuzzy left (right, lateral) ideals in ternary semigroups and characterized regular and intra-regular ternary semigroups by using the concept of fuzzy ideals of ternary semigroups. Our main aim in this study is to use the idea of Jun and Kar to introduce the notion of hesitant fuzzy ideals in ternary semigroups and study their related properties. Here we define hesitant fuzzy left (right, lateral) ideals of ternary semigroups and characterize regular and intra-regular ternary semigroups by using the concept of hesitant fuzzy ideals of ternary semigroups.

Definition 1. [5] A non-empty set $S$ with a ternary operation $S \times S \times$ $S \rightarrow S$, written as $\left(x_{1}, x_{2}, x_{3}\right) \mapsto\left[x_{1}, x_{2}, x_{3}\right]$, is called a ternary semigroup if it satisfies the following identity, for any $x_{1}, x_{2}, x_{3}, x_{4}, x_{5} \in S$,

$$
\left[\left[x_{1} x_{2} x_{3}\right] x_{4} x_{5}\right]=\left[x_{1}\left[x_{2} x_{3} x_{4}\right] x_{5}\right]=\left[\left[x_{1} x_{2}\left[x_{3} x_{4} x_{5}\right]\right] .\right.
$$

Example 2. Let $S=\left\{\left(\begin{array}{lll}0 & 0 & a \\ 0 & b & 0 \\ c & 0 & 0\end{array}\right): a, b, c \in \mathbb{N} \cup\{0\}\right\}$. Then $S$ is a ternary semigroup under the usual multiplication of matrices over $\mathbb{N} \cup\{0\}$ while $S$ is not a semigroup under the same operation.

If $A=\{a\}$, then we write $[\{a\} B C]$ as $[a B C]$ and similarly if $B=\{b\}$ or $C=\{c\}$, we write $[A b C]$ and $[A B c]$, respectively. For the sake of simplicity, we write $\left[x_{1} x_{2} x_{3}\right]$ as $x_{1} x_{2} x_{3}$ and $[A B C]$ as $A B C$.

Definition 3. A non-empty subset $T$ of a ternary semigroup $S$ is called a ternary subsemigroup of $S$ if $T T T \subseteq T$.

Definition 4. An element $a$ in a ternary semigroup $S$ is called regular if there exists an element $x$ in $S$ such that $a x a=a$.

Torra defined HFSs in terms of a function that returns a set of membership values for each element in the domain. We give some background on the basic notions on HFSs. For more details, we refer to references.

Let $S$ be a reference set. Then we define HFS on $S$ in terms of a function 
$\mathcal{H}$ that when applied to $X$ returns a subset of $[0,1]$. For a HFS $\mathcal{H}$ on $S$ and $x$, $y \in S$, we use the notations $\mathcal{H}_{x}:=\mathcal{H}(x)$ and $\mathcal{H}_{x}=\mathcal{H}_{y} \Leftrightarrow \mathcal{H}_{x} \subseteq \mathcal{H}_{y}, \mathcal{H}_{x} \supseteq \mathcal{H}_{y}$.

Let $\mathcal{H}$ and $\mathcal{G}$ be two HFSs on $S$. The hesitant union $\mathcal{H} \sqcup \mathcal{G}$ and hesitant intersection $\mathcal{H} \sqcap \mathcal{G}$ of $\mathcal{H}$ and $\mathcal{G}$ are defined to be HFSs on $S$ by:

$$
\mathcal{H} \sqcup \mathcal{G}: S \rightarrow \mathbf{P}([0,1]), x \mapsto \mathcal{H}_{x} \cup \mathcal{G}_{x}
$$

and

$$
\mathcal{H} \sqcap \mathcal{G}: S \rightarrow \mathbf{P}([0,1]), x \mapsto \mathcal{H}_{x} \cap \mathcal{G}_{x}
$$

For any HFSs $\mathcal{H}$ and $\mathcal{G}$ on $S$, we define

$$
\mathcal{H} \sqsubseteq \mathcal{G} \text { if } \mathcal{H}_{x} \subseteq \mathcal{G}_{x} \text { for all } x \in S .
$$

For a non-empty subset $A$ of $S$ and $\varepsilon, \delta \in \mathbf{P}([0,1])$ with $\varepsilon \supsetneq \delta$, define a map $\left[\chi_{A}^{(\varepsilon, \delta)}\right]$ as follows:

$$
\left[\chi_{A}^{(\varepsilon, \delta)}\right]: S \rightarrow \mathbf{P}([0,1]), x \mapsto \begin{cases}\varepsilon, & \text { if } x \in A, \\ \delta, & \text { otherwise }\end{cases}
$$

Then $\left[\chi_{A}^{(\varepsilon, \delta)}\right]$ is a HFS on $S$, which is called the $(\varepsilon, \delta)$-characteristic HFS. The HFS $\left[\chi_{S}^{(\varepsilon, \delta)}\right]$ is called the $(\varepsilon, \delta)$-identity HFS on $S$. The $(\varepsilon, \delta)$-characteristic HFS with $\varepsilon=[0,1]$ and $\delta=\emptyset$ is called the characteristic HFS, and is denoted by $\left[\chi_{A}\right]$. The $(\varepsilon, \delta)$-identity HFS with $\varepsilon=[0,1]$ and $\delta=\emptyset$ is called the identity HFS, and is denoted by $\left[\chi_{S}\right]$.

\section{Hesitant Fuzzy Ideals on Ternary Semigroups}

Definition 5. A non-empty hesitant fuzzy subset $\mathcal{H}$ of $S$ is called a hesitant fuzzy ternary subsemigroup on $S$ if $\mathcal{H}_{u v w} \supseteq \mathcal{H}_{u} \cap \mathcal{H}_{v} \cap \mathcal{H}_{w}$ for all $u$, $v, w \in S$.

Definition 6. A non-empty hesitant fuzzy subset $\mathcal{H}$ of $S$ is called a hesitant fuzzy left (resp. lateral, right) ideal on $S$ if $\mathcal{H}_{u v w} \supseteq \mathcal{H}_{w}\left(\mathcal{H}_{u v w} \supseteq \mathcal{H}_{v}\right.$, $\mathcal{H}_{u v w} \supseteq \mathcal{H}_{u}$ ) for all $u, v, w \in S$. 
Example 7. Let $S=\left\{O=\left(\begin{array}{lll}0 & 0 & 0 \\ 0 & 0 & 0 \\ 0 & 0 & 0\end{array}\right), X=\left(\begin{array}{lll}0 & 0 & 1 \\ 0 & 0 & 0 \\ 0 & 0 & 0\end{array}\right)\right.$, $\left.Y=\left(\begin{array}{lll}0 & 0 & 0 \\ 0 & 1 & 0 \\ 0 & 0 & 0\end{array}\right), Z=\left(\begin{array}{lll}0 & 0 & 0 \\ 0 & 0 & 0 \\ 1 & 0 & 0\end{array}\right), W=\left(\begin{array}{lll}0 & 0 & 1 \\ 0 & 1 & 0 \\ 1 & 0 & 0\end{array}\right)\right\}$. Then $S$ is a ternary semigroup under the usual multiplication of matrices.

Consider $L=\{O, Z\}$. Then $L$ is a left ideal of ternary semigroup $S$.

Now we define a HFS $\mathcal{L}$ on $S$ by:

$$
\mathcal{L}: S \rightarrow \mathbf{P}[0,1], \quad x \mapsto\left\{\begin{array}{cl}
(0.7,0.9], & \text { if } x=\text { O or } Z, \\
\emptyset, & \text { if } x=X \text { or } Y \text { or } W
\end{array}\right.
$$

Hence we can easily show that $\mathcal{L}$ is a hesitant fuzzy left ideal on $S$.

We say that $\mathcal{H}$ is a hesitant fuzzy two sided ideal if $\mathcal{H}$ is both a hesitant fuzzy left ideal and a hesitant fuzzy right ideal on $S$. $\mathcal{H}$ is called a hesitant fuzzy ideal if $\mathcal{H}$ is a hesitant fuzzy left ideal, a hesitant fuzzy lateral ideal and a hesitant fuzzy right ideal on $S$.

Lemma 8. Let $S$ be a ternary semigroup. If $A$ is a non-empty subset and $\left[\chi_{A}^{(\varepsilon, \delta)}\right]$ is a $(\varepsilon, \delta)$-characteristic HFS on $S$. Then,

(1) $A$ is a ternary subsemigroup on $S$ if and only if $\left[\chi_{A}^{(\varepsilon, \delta)}\right]$ is a hesitant fuzzy ternary subsemigroup on $S$.

(2) $A$ is a left(resp. lateral, right) ideal on $S$ if and only if $\left[\chi_{A}^{(\varepsilon, \delta)}\right]$ is a hesitant fuzzy left(resp. lateral, right)ideal on $S$.

Proof. The proof is a straightforward.

Definition 9. Let $\mathcal{H}, \mathcal{I}, \mathcal{K} \in H F(S)$, where $H F(S)$ is the set of all HFS on $S$. The product of $\mathcal{H}, \mathcal{I}, \mathcal{K}$ denoted by $\mathcal{H} \hat{\diamond} \mathcal{I} \hat{\diamond} \mathcal{K}$ is defined as, for any $x$ $\in S$

$$
\begin{aligned}
& (\mathcal{H} \hat{\diamond} \mathcal{I} \hat{\diamond} \mathcal{K})_{x}= \\
& \left\{\begin{array}{c}
\bigcup_{x=u v w}\left\{\mathcal{H}_{u} \cap \mathcal{I}_{v} \cap \mathcal{K}_{w}\right\} \quad \text { if } \exists u, v, w \in S \text { s.t. } x=\text { uvw } \\
\emptyset \quad \text { otherwise. }
\end{array}\right.
\end{aligned}
$$


Lemma 10. Let $\mathcal{H} \in H F(S)$ such that $\mathcal{H}_{u} \subseteq \varepsilon$ for all $u \in S$ and $\left[\chi_{S}^{(\varepsilon, \delta)}\right]$ be a $(\varepsilon, \delta)$-identity HFS on $S$. Then,

(1) $\mathcal{H}$ is a hesitant fuzzy ternary subsemigroup on $S$ if and only if $\mathcal{H} \hat{\diamond} \mathcal{H} \hat{\diamond}$ $\mathcal{H} \sqsubseteq \mathcal{H}$.

(2) $\mathcal{H}$ is a hesitant fuzzy left ideal on $S$ if and only if $\left[\chi_{S}^{(\varepsilon, \delta)}\right] \hat{\diamond}\left[\chi_{S}^{(\varepsilon, \delta)}\right] \hat{\diamond} \mathcal{H}$ $\sqsubseteq \mathcal{H}$

(3) $\mathcal{H}$ is a hesitant fuzzy lateral ideal on $S$ if and only if $\left[\chi_{S}^{(\varepsilon, \delta)}\right] \hat{\diamond} \mathcal{H} \hat{\diamond}\left[\chi_{S}^{(\varepsilon, \delta)}\right]$ $\sqsubseteq \mathcal{H}$ $\sqsubseteq \mathcal{H}$

(4) $\mathcal{H}$ is a hesitant fuzzy right ideal on $S$ if and only if $\mathcal{H} \hat{\diamond}\left[\chi_{S}^{(\varepsilon, \delta)}\right] \hat{\diamond}\left[\chi_{S}^{(\varepsilon, \delta)}\right]$

Proof. (1) Let $\mathcal{H}$ be a hesitant fuzzy ternary subsemigroup on $S$ and $x \in$ $S$. Now we have following two cases:

Case 1: If $h \neq u v w$ for any $u, v, w \in S$, then $(\mathcal{H} \hat{\diamond} \mathcal{H} \hat{\diamond} \mathcal{H})_{h}=\emptyset \subseteq \mathcal{H}_{h}$.

Case 2: If there exists $h$ such that $h=u v w$ for some $u, v, w \in S$, then

$$
\begin{aligned}
(\mathcal{H} \hat{\diamond} \mathcal{H} \hat{\diamond} \mathcal{H})_{h} & =\bigcup_{h=u v w}\left\{\mathcal{H}_{u} \cap \mathcal{H}_{v} \cap \mathcal{H}_{w}\right\} \\
& \subseteq \bigcup_{h=u v w}\left\{\mathcal{H}_{u v w}\right\} \\
& =\bigcup_{h=u v w}\left\{\mathcal{H}_{h}\right\} \\
& =\mathcal{H}_{h} .
\end{aligned}
$$

Hence in all the cases $\mathcal{H} \hat{\diamond} \mathcal{H} \hat{\diamond} \mathcal{H} \sqsubseteq \mathcal{H}$.

Conversely, suppose $\mathcal{H} \hat{\diamond} \mathcal{H} \hat{\diamond} \mathcal{H} \sqsubseteq \mathcal{H}$ for any $\mathcal{H} \in H F(S)$ and let $u, v, w \in$ $S$. As $S$ is a ternary semigroup, we have uvw $\in S$. Let $h=u v w$. Then,

$$
\begin{aligned}
\mathcal{H}_{\text {uvw }} & =\mathcal{H}_{h} \\
& \supseteq(\mathcal{H} \hat{\diamond} \mathcal{H} \hat{\diamond} \mathcal{H})_{h} \\
& =\bigcup_{h=r s t}\left\{\mathcal{H}_{r} \cap \mathcal{H}_{s} \cap \mathcal{H}_{t}\right\} \\
& \supseteq \mathcal{H}_{u} \cap \mathcal{H}_{v} \cap \mathcal{H}_{w} .
\end{aligned}
$$

Therefore $\mathcal{H}$ is a hesitant fuzzy ternary subsemigroup on $S$.

(2) Let $\mathcal{H}$ be a hesitant fuzzy left ideal on $S$ and $h \in S$. Then we have following two cases:

Case 1: If $h \neq u v w$ for any $u, v, w \in S$, then $\left(\left[\chi_{S}^{(\varepsilon, \delta)}\right] \hat{\diamond}\left[\chi_{S}^{(\varepsilon, \delta)}\right] \hat{\diamond} \mathcal{H}\right)_{h}=\emptyset$ $\subseteq \mathcal{H}_{h}$ 
Case 2: If there exists $h$ such that $h=u v w$ for some $u, v, w \in S$, then

$$
\begin{aligned}
\left(\left[\chi_{S}^{(\varepsilon, \delta)}\right] \hat{\diamond}\left[\chi_{S}^{(\varepsilon, \delta)}\right] \hat{\diamond} \mathcal{H}\right)_{h} & =\bigcup_{h=u v w}\left\{\left[\chi_{S}^{(\varepsilon, \delta)}\right]_{u} \cap\left[\chi_{S}^{(\varepsilon, \delta)}\right]_{v} \cap \mathcal{H}_{w}\right\} \\
& =\bigcup_{h=u v w}\left\{\varepsilon \cap \varepsilon \cap \mathcal{H}_{w}\right\} \\
& =\bigcup_{h=u v w}\left\{\mathcal{H}_{w}\right\} \text { as }\left(\left[\chi_{S}^{(\varepsilon, \delta)}\right]_{u}=\left[\chi_{S}^{(\varepsilon, \delta)}\right]_{v}=\varepsilon\right) \\
& \subseteq \bigcup_{h=u v w}\left\{\mathcal{H}_{u v w}\right\} \\
& =\bigcup_{h=u v w}\left\{\mathcal{H}_{h}\right\} \\
& =\mathcal{H}_{h} .
\end{aligned}
$$

Therefore $\mathcal{H} \hat{\diamond} \mathcal{H} \hat{\diamond} \mathcal{H} \sqsubseteq \mathcal{H}$

Conversely, suppose $\left[\chi_{S}^{(\varepsilon, \delta)}\right] \hat{\diamond}\left[\chi_{S}^{(\varepsilon, \delta)}\right] \hat{\diamond} \mathcal{H} \sqsubseteq \mathcal{H}$ for any $\mathcal{H} \in H F(S)$ and let $u, v, w \in S$. As $S$ is a ternary semigroup, we have $u v w \in S$. Let $h=u v w$. Then,

$$
\begin{aligned}
\mathcal{H}_{u v w} & =\mathcal{H}_{h} \\
& \supseteq\left(\left[\chi_{S}^{(\varepsilon, \delta)}\right] \hat{\diamond}\left[\chi_{S}^{(\varepsilon, \delta)}\right] \hat{\diamond} \mathcal{H}\right)_{h} \\
& =\bigcup_{h=r s t}\left\{\left[\chi_{S}^{(\varepsilon, \delta)}\right]_{r} \cap\left[\chi_{S}^{(\varepsilon, \delta)}\right]_{s} \cap \mathcal{H}_{t}\right\} \\
& \supseteq\left[\chi_{S}^{(\varepsilon, \delta)}\right]_{u} \cap\left[\chi_{S}^{(\varepsilon, \delta)}\right]_{v} \cap \mathcal{H}_{w} \\
& =\mathcal{H}_{w}\left(A s\left[\chi_{S}^{(\varepsilon, \delta)}\right]_{u}=\left[\chi_{S}^{(\varepsilon, \delta)}\right]_{v}=\varepsilon\right) .
\end{aligned}
$$

Hence $\mathcal{H}$ is a hesitant fuzzy left ideal on $S$.

(3) and (4) are analogous to (2).

Example 11. Consider $S$ as given in the Example 7. Let $\left[\chi_{S}^{(\varepsilon, \delta)}\right]$ be a $(\varepsilon, \delta)$-identity HFS on $S$, where $\varepsilon=[0,1], \delta=\emptyset$. Now we define HFS $\mathcal{R}$ on $S$ by:

$$
\mathcal{R}: S \rightarrow \mathbf{P}[0,1], \quad x \mapsto\left\{\begin{array}{cc}
(0.5,0.8], & \text { if } x=O \text { or } X, \\
\emptyset, & \text { if } x=Y \text { or } Z \text { or } W
\end{array}\right.
$$

Then it is easy to verify that $\mathcal{R} \hat{\diamond}\left[\chi_{S}^{(\varepsilon, \delta)}\right] \hat{\diamond}\left[\chi_{S}^{(\varepsilon, \delta)}\right] \sqsubseteq \mathcal{R}$. Hence $\mathcal{R}$ is a hesitant fuzzy right ideal on $S$.

Example 12. Consider $S$ as given in the Example 7. Let $\left[\chi_{S}^{(\varepsilon, \delta)}\right]$ be a $(\varepsilon, \delta)$-identity HFS on $S$, where $\varepsilon=[0,1], \delta=\emptyset$. Now we define HFS $\mathcal{M}$ on $S$ by: 


$$
\mathcal{M}: S \rightarrow \mathbf{P}[0,1], \quad x \mapsto\left\{\begin{array}{cl}
(0.7,0.8], & \text { if } x=O, \\
(0.5,0.65], & \text { if } x=X \text { or } Y \\
\emptyset, & \text { if } x=Z \text { or } W
\end{array} .\right.
$$

For $Z \in S, \mathcal{M}_{Z}=\emptyset$. Now

$$
Z=\left(\begin{array}{lll}
0 & 0 & 0 \\
0 & 0 & 0 \\
1 & 0 & 0
\end{array}\right)=\left(\begin{array}{lll}
0 & 0 & 1 \\
0 & 1 & 0 \\
1 & 0 & 0
\end{array}\right)\left(\begin{array}{lll}
0 & 0 & 1 \\
0 & 0 & 0 \\
0 & 0 & 0
\end{array}\right)\left(\begin{array}{lll}
0 & 0 & 1 \\
0 & 1 & 0 \\
1 & 0 & 0
\end{array}\right) .
$$

It implies $Z=W X W$. Hence we have

$$
\begin{aligned}
\left(\left[\chi_{S}^{(\varepsilon, \delta)}\right] \hat{\diamond} \mathcal{M} \hat{\diamond}\left[\chi_{S}^{(\varepsilon, \delta)}\right]\right)_{Z} & =\bigcup_{Z=U V P}\left\{\left[\chi_{S}^{(\varepsilon, \delta)}\right]_{U} \cap \mathcal{M}_{V} \cap\left[\chi_{S}^{(\varepsilon, \delta)}\right]_{P}\right\} \\
& =\left\{\left[\chi_{S}^{(\varepsilon, \delta)}\right]_{W} \cap \mathcal{M}_{X} \cap\left[\chi_{S}^{(\varepsilon, \delta)}\right]_{W}\right\} \\
& =\varepsilon \cap(0.5,0.65] \cap \varepsilon \\
& =(0.5,0.65] .
\end{aligned}
$$

Then it can be seen that $\left(\left[\chi_{S}^{(\varepsilon, \delta)}\right] \hat{\diamond} \mathcal{M} \hat{\diamond}\left[\chi_{S}^{(\varepsilon, \delta)}\right]\right)_{Z} \nsubseteq \mathcal{M}_{Z}$. Hence $\left[\chi_{S}^{(\varepsilon, \delta)}\right] \hat{\diamond} \mathcal{M} \hat{\diamond}$ $\left[\chi_{S}^{(\varepsilon, \delta)}\right] \operatorname{not} \sqsubseteq \mathcal{M}$. Therefore $\mathcal{M}$ is not a hesitant fuzzy lateral ideal on $S$.

Lemma 13. If $\mathcal{H}$ and $\mathcal{G}$ are two hesitant fuzzy ternary subsemigroups on $S$, then $\mathcal{H} \sqcap \mathcal{G}$ is also a hesitant fuzzy ternary subsemigroup on $S$.

Proof. The proof is a straightforward.

Lemma 14. If $\mathcal{H}$ and $\mathcal{G}$ are two hesitant fuzzy left(resp. lateral, right) ideals on $S$ and $\left[\chi_{S}^{(\varepsilon, \delta)}\right]$ be a $(\varepsilon, \delta)$-identity HFS on $S$. Then $\mathcal{H} \sqcap \mathcal{G}$ is also a hesitant fuzzy left (resp. lateral, right) ideal on $S$.

Proof. The proof is a straightforward.

Definition 15. Let $S$ be a non-empty set and $h \in S$. Let $\varepsilon \subseteq[0,1]$ such that $\varepsilon \neq \emptyset$. A hesitant fuzzy point $\bar{h}_{\varepsilon}$ on $S$ is a hesitant fuzzy subset of $S$ defined by:

$$
\left(\bar{h}_{\varepsilon}\right)_{x}=\left\{\begin{array}{ll}
\varepsilon, & \text { if } x=h, \\
\emptyset, & \text { otherwise }
\end{array} \quad, \text { where } x \in S\right.
$$


We will denote the set of all hesitant fuzzy points by $\operatorname{HFP}(S)$.

Proposition 16. Let $\alpha, \varepsilon, \beta \in \mathbf{P}([0,1])$ and $\bar{g}_{\alpha}, \bar{h}_{\varepsilon}, \bar{i}_{\beta} \in \operatorname{HFP}(S)$ such

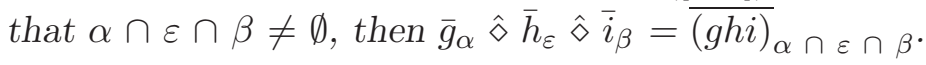

Proof. The proof is a straightforward.

Proposition 17. Let $\bar{h}_{\alpha} \in \operatorname{HFP}(S)$ such that $\alpha \subseteq \varepsilon$. Then for any $x \in$ $S$,

(1) $\left(\left[\chi_{S}^{(\varepsilon, \delta)}\right] \hat{\diamond}\left[\chi_{S}^{(\varepsilon, \delta)}\right] \hat{\diamond} \bar{h}_{\alpha}\right)_{x}=\left\{\begin{array}{cc}\alpha, & \text { if } x \in S S h, \\ \emptyset, & \text { otherwise. }\end{array}\right.$
(2) $\left(\left[\chi_{S}^{(\varepsilon, \delta)}\right] \hat{\diamond} \bar{h}_{\alpha} \hat{\diamond}\left[\chi_{S}^{(\varepsilon, \delta)}\right]\right)_{x}=\left\{\begin{array}{rr}\alpha, & \text { if } x \in S h S, \\ \emptyset, & \text { otherwise. }\end{array}\right.$
(3) $\left(\bar{h}_{\alpha} \hat{\diamond}\left[\chi_{S}^{(\varepsilon, \delta)}\right] \hat{\diamond}\left[\chi_{S}^{(\varepsilon, \delta)}\right]\right)_{x}=\left\{\begin{array}{cc}\alpha, & \text { if } x \in h S S, \\ \emptyset, & \text { otherwise. }\end{array}\right.$

Proof. (1) Let $x \in S$. We have following two cases:

Case 1: If $x \neq r s t$ for any $r, s, t \in S$, then $\left(\left[\chi_{S}^{(\varepsilon, \delta)}\right] \hat{\diamond}\left[\chi_{S}^{(\varepsilon, \delta)}\right] \hat{\diamond} \bar{h}_{\alpha}\right)_{x}=\emptyset$.

Case 2: If $x=r$ st for some $r, s, t \in S$, then

$$
\left(\left[\chi_{S}^{(\varepsilon, \delta)}\right] \hat{\diamond}\left[\chi_{S}^{(\varepsilon, \delta)}\right] \hat{\diamond} \bar{h}_{\alpha}\right)_{x}=\bigcup_{x=r s t}\left\{\left[\chi_{S}^{(\varepsilon, \delta)}\right]_{r} \cap\left[\chi_{S}^{(\varepsilon, \delta)}\right]_{s} \cap\left(\bar{h}_{\alpha}\right)_{t}\right\} .
$$

Subcase 1: If $x \neq u v h$ for any $u, v \in S$, then $\left(\left[\chi_{S}^{(\varepsilon, \delta)}\right] \hat{\diamond}\left[\chi_{S}^{(\varepsilon, \delta)}\right] \hat{\diamond} \bar{h}_{\alpha}\right)_{x}=\emptyset$.

Subcase 2: If there exist $x$ such that $x=u v h$ for some $u, v \in S$, then $x$ $\in S S h$ and hence

$$
\begin{aligned}
\left(\left[\chi_{S}^{(\varepsilon, \delta)}\right] \hat{\diamond}\left[\chi_{S}^{(\varepsilon, \delta)}\right] \hat{\diamond} \bar{h}_{\alpha}\right)_{x} & =\bigcup_{x=r s t}\left\{\left[\chi_{S}^{(\varepsilon, \delta)}\right]_{r} \cap\left[\chi_{S}^{(\varepsilon, \delta)}\right]_{s} \cap\left(\bar{h}_{\alpha}\right)_{t}\right\} . \\
& =\left[\chi_{S}^{(\varepsilon, \delta)}\right]_{u} \cap\left[\chi_{S}^{(\varepsilon, \delta)}\right]_{v} \cap\left(\bar{h}_{\alpha}\right)_{h} \\
& =\varepsilon \cap \varepsilon \cap \alpha \\
& =\alpha .
\end{aligned}
$$

Hence the result is proved.

Proofs of (2) and (3) are analogous to (1). 
Corollary 18. Let $\bar{h}_{\alpha} \in \operatorname{HFP}(S)$, then for any $x \in S$,

$$
\left(\bar{h}_{\alpha} \sqcup\left(\left[\chi_{S}^{(\varepsilon, \delta)}\right] \hat{\diamond}\left[\chi_{S}^{(\varepsilon, \delta)}\right] \hat{\diamond} \bar{h}_{\alpha}\right)\right)_{x}= \begin{cases}\alpha, & \text { if } x=h \text { or } x \in S S h, \\ \emptyset, & \text { otherwise. }\end{cases}
$$

Proof. It is easy to show that $\bar{h}_{\alpha} \sqcup\left(\left[\chi_{S}^{(\varepsilon, \delta)}\right] \hat{\diamond}\left[\chi_{S}^{(\varepsilon, \delta)}\right] \hat{\diamond} \bar{h}_{\alpha}\right)$ is a hesitant fuzzy left ideal on $S$ containing $\bar{h}_{\alpha}$. If $x=h$, then $\left(\bar{h}_{\alpha} \sqcup\left(\left[\chi_{S}^{(\varepsilon, \delta)}\right] \hat{\diamond}\left[\chi_{S}^{(\varepsilon, \delta)}\right] \hat{\diamond} \bar{h}_{\alpha}\right)\right)_{x}=$ $\left(\bar{h}_{\alpha}\right)_{h} \cup\left(\left[\chi_{S}^{(\varepsilon, \delta)}\right] \hat{\diamond}\left[\chi_{S}^{(\varepsilon, \delta)}\right] \hat{\diamond} \bar{h}_{\alpha}\right)_{h}=\alpha \cup \emptyset=\alpha$.

If $x=u v h \in S$ for some $u, v \in S$, then $\left(\bar{h}_{\alpha} \sqcup\left(\left[\chi_{S}^{(\varepsilon, \delta)}\right] \hat{\diamond}\left[\chi_{S}^{(\varepsilon, \delta)}\right] \hat{\diamond} \bar{h}_{\alpha}\right)\right)_{x}=$ $\left(\bar{h}_{\alpha}\right)_{u v h} \cup\left(\left[\chi_{S}^{(\varepsilon, \delta)}\right] \hat{\diamond}\left[\chi_{S}^{(\varepsilon, \delta)}\right] \hat{\diamond} \bar{h}_{\alpha}\right)_{u v h}=\emptyset \cup \alpha($ by the Proposition $17(1))=\alpha$.

If $x \neq h$ and $x \neq u v h$ for any $u, v \in S$, then $\left(\bar{h}_{\alpha} \sqcup\left(\left[\chi_{S}^{(\varepsilon, \delta)}\right] \hat{\diamond}\left[\chi_{S}^{(\varepsilon, \delta)}\right] \hat{\diamond} \bar{h}_{\alpha}\right)\right)_{x}$ $=\emptyset$. Hence proof the result.

Definition 19. Let $\mathcal{H} \in H F(S)$ and $\bar{h}_{\alpha} \in H F P(S)$. Then we say $\bar{h}_{\alpha} \in$ $\mathcal{H}$ if $(\mathcal{H})_{h} \supseteq \alpha$.

Proposition 20. For any $\mathcal{H} \in H F(S)$ and $\bar{h}_{\alpha}, \bar{g}_{\beta} \in H F P(S)$. Then,

(1) $\bar{h}_{\alpha} \in \mathcal{H}$ if and only if $\bar{h}_{\alpha} \sqsubseteq \mathcal{H}$.

(2) $\mathcal{H}=\bigcup_{\bar{h}_{\alpha} \in \mathcal{H}} \bar{h}_{\alpha}$.

(3) $\bar{h}_{\alpha} \sqsubseteq \bar{g}_{\beta}$ if and only if $h=g$ and $\alpha \subseteq \beta$.

(4) $\bar{h}_{\alpha} \hat{\diamond} \mathcal{H} \hat{\diamond} \bar{g}_{\beta}=\bigcup_{\bar{z}_{\gamma} \in \mathcal{H}} \bar{h}_{\alpha} \hat{\diamond} \bar{z}_{\gamma} \hat{\diamond} \bar{g}_{\beta}$.

Definition 21. (1) Let $S$ be a ternary semigroup and $\bar{h}_{\alpha}$ be a hesitant fuzzy point on $S$. Then the hesitant intersection of all hesitant fuzzy left ideals on $S$ containing $\bar{h}_{\alpha}$ is a hesitant fuzzy left ideal on $S$ containing $\bar{h}_{\alpha}$, denoted by $<\bar{h}_{\alpha}>_{L}$ and defined as

$$
<\bar{h}_{\alpha}>_{L}=\prod_{\bar{h}_{\alpha} \in \mathcal{H} \in H F L_{L}(S)} \mathcal{H},
$$

where $\operatorname{HFL}_{L}(S)$ is the set of all hesitant fuzzy left ideals on $S .<\bar{h}_{\alpha}>_{L}$ is said to be hesitant fuzzy left ideal generated by hesitant fuzzy point $\bar{h}_{\alpha}$.

Analogously, we can define $<\bar{h}_{\alpha}>_{M}$ and $<\bar{h}_{\alpha}>_{R}$ respectively, the hesitant fuzzy lateral ideal and hesitant fuzzy right ideal generated by the hesitant fuzzy point $\bar{h}_{\alpha}$.

Proposition 22. Let $\bar{h}_{\alpha} \in H F P(S)$. For any $x \in S$, 


$$
\left(<\bar{h}_{\alpha}>_{L}\right)_{x}= \begin{cases}\alpha, & \text { if } x \in<h>_{L}, \\ \emptyset, & \text { otherwise }\end{cases}
$$

Proof. As we know that $\left\langle\bar{h}_{\alpha}>_{L}=\prod_{\bar{h}_{\alpha} \in \mathcal{H} \in H F L_{L}(S)} \mathcal{H}\right.$. Hence for any $x \in$ $S,\left(<\bar{h}_{\alpha}>_{L}\right)_{x}=\left(\prod_{\bar{h}_{\alpha} \in \mathcal{H} \in H F L_{L}(S)} \mathcal{H}\right)_{x}=\left(\bigcap_{\bar{h}_{\alpha} \in \mathcal{H} \in H F L_{L}(S)}\right) \mathcal{H}_{x}$.

If $x=h$, then $\left(<\bar{h}_{\alpha}>_{L}\right)_{x}=\left(<\bar{h}_{\alpha}>_{L}\right)_{h}=\left(\bigcap_{\bar{h}_{\alpha} \in \mathcal{H} \in H F L_{L}(S)}\right)(\mathcal{H})_{h}=\alpha($ As $\left.\bar{h}_{\alpha} \in \mathcal{H},(\mathcal{H})_{h} \supseteq \alpha\right)$.

If $x=u v h$ for some $u, v \in S$, then for any hesitant fuzzy left ideal $\mathcal{H}$ on $S$, $\mathcal{H}_{u v h} \supseteq \mathcal{H}_{h} \supseteq \alpha$ and therefore $\left(<\bar{h}_{\alpha}>_{L}\right)_{x}=\left(\bigcap_{\bar{h}_{\alpha} \in \mathcal{H} \in H F L_{L}(S)}\right)(\mathcal{H})_{u v h}=\alpha$.

If $x \neq h$ and $x \neq u v h$ for any $u, v \in S$, then by the Corollary 18, we have $\left(<\bar{h}_{\alpha}>_{L}\right)_{x}=\emptyset$. Hence the result is proved.

Proposition 23. Let $\bar{h}_{\alpha} \in H F P(S)$. For any $x \in S$,
(1) $\left(<\bar{h}_{\alpha}>_{M}\right)_{x}= \begin{cases}\alpha, & \text { if } x \in<h>_{M}, \\ \emptyset, & \text { otherwise. }\end{cases}$
(2) $\left(<\bar{h}_{\alpha}>_{R}\right)_{x}= \begin{cases}\alpha, & \text { if } x \in<h>_{R}, \\ \emptyset, & \text { otherwise }\end{cases}$

Proof. Proofs of (1) and (2) are analogous to Proposition 22.

Proposition 24. Let $\bar{h}_{\alpha} \in H F P(S)$. Then,

$$
\begin{aligned}
& (1)<\bar{h}_{\alpha}>_{L}=\bar{h}_{\alpha} \sqcup\left(\left[\chi_{S}^{(\varepsilon, \delta)}\right] \hat{\diamond}\left[\chi_{S}^{(\varepsilon, \delta)}\right] \hat{\diamond} \bar{h}_{\alpha}\right) . \\
& (2)<\bar{h}_{\alpha}>_{M}=\bar{h}_{\alpha} \sqcup\left(\left[\chi_{S}^{(\varepsilon, \delta)}\right] \hat{\diamond} \bar{h}_{\alpha} \hat{\diamond}\left[\chi_{S}^{(\varepsilon, \delta)}\right]\right) \sqcup\left(\left[\chi_{S}^{(\varepsilon, \delta)}\right] \hat{\diamond}\left[\chi_{S}^{(\varepsilon, \delta)}\right] \hat{\diamond} \bar{h}_{\alpha}\right. \\
& \left.\hat{\diamond}\left[\chi_{S}^{(\varepsilon, \delta)}\right] \hat{\diamond}\left[\chi_{S}^{(\varepsilon, \delta)}\right]\right) . \\
& (3)<\bar{h}_{\alpha}>_{R}=\bar{h}_{\alpha} \sqcup\left(\bar{h}_{\alpha} \hat{\diamond}\left[\chi_{S}^{(\varepsilon, \delta)}\right] \hat{\diamond}\left[\chi_{S}^{(\varepsilon, \delta)}\right]\right) .
\end{aligned}
$$

Proof. It is easy to show that $\bar{h}_{\alpha} \sqcup\left(\left[\chi_{S}^{(\varepsilon, \delta)}\right] \hat{\diamond}\left[\chi_{S}^{(\varepsilon, \delta)}\right] \hat{\diamond} \bar{h}_{\alpha}\right)$ is a hesitant fuzzy left ideal on $S$ containing $\bar{h}_{\alpha}$. Now it remains to show that it is the smallest hesitant fuzzy left ideal on $S$ containing $\bar{h}_{\alpha}$. For this let $\mathcal{G}$ be another hesitant fuzzy left ideal on $S$ containing $\bar{h}_{\alpha}$ such that $\mathcal{G} \sqsubseteq \bar{h}_{\alpha} \sqcup\left(\left[\chi_{S}^{(\varepsilon, \delta)}\right] \hat{\diamond}\left[\chi_{S}^{(\varepsilon, \delta)}\right] \hat{\diamond} \bar{h}_{\alpha}\right)$. As $\bar{h}_{\alpha} \in \mathcal{G}$, we have $\left[\chi_{S}^{(\varepsilon, \delta)}\right] \hat{\diamond}\left[\chi_{S}^{(\varepsilon, \delta)}\right] \hat{\diamond} \bar{h}_{\alpha} \sqsubseteq\left[\chi_{S}^{(\varepsilon, \delta)}\right] \hat{\diamond}\left[\chi_{S}^{(\varepsilon, \delta)}\right] \hat{\diamond} \mathcal{G} \sqsubseteq \mathcal{G}$ (As $\mathcal{G}$ is a hesitant fuzzy left ideal on $S)$. It implies $\bar{h}_{\alpha} \sqcup\left(\left[\chi_{S}^{(\varepsilon, \delta)}\right] \hat{\diamond}\left[\chi_{S}^{(\varepsilon, \delta)}\right] \hat{\diamond} \bar{h}_{\alpha}\right)$ $\sqsubseteq \mathcal{G}$. Therefore $\mathcal{G}=\bar{h}_{\alpha} \sqcup\left(\left[\chi_{S}^{(\varepsilon, \delta)}\right] \hat{\diamond}\left[\chi_{S}^{(\varepsilon, \delta)}\right] \hat{\diamond} \bar{h}_{\alpha}\right)$. This shows that $\bar{h}_{\alpha} \sqcup$ 
$\left(\left[\chi_{S}^{(\varepsilon, \delta)}\right] \hat{\diamond}\left[\chi_{S}^{(\varepsilon, \delta)}\right] \hat{\diamond} \bar{h}_{\alpha}\right)$ is a smallest hesitant fuzzy left ideal on $S$ containing hesitant fuzzy point $\bar{h}_{\alpha}$. Therefore $<\bar{h}_{\alpha}>_{L}=\bar{h}_{\alpha} \sqcup\left(\left[\chi_{S}^{(\varepsilon, \delta)}\right] \hat{\diamond}\left[\chi_{S}^{(\varepsilon, \delta)}\right] \hat{\diamond} \bar{h}_{\alpha}\right)$.

Proofs of (2) and (3) are analogous to (1).

Definition 25. A ternary semigroup $S$ is called regular if for each element $a$ of $S$, there exists an element $x$ in $S$ such that $a x a=a$.

Theorem 26. Let $S$ be a ternary semigroup. Then the following statements are equivalent:

(1) $S$ is regular;

(2) $\mathcal{R} \hat{\diamond} \mathcal{M} \hat{\diamond} \mathcal{L}=\mathcal{R} \sqcap \mathcal{M} \sqcap \mathcal{L}$, for every hesitant fuzzy right ideal $\mathcal{R}$, hesitant fuzzy lateral ideal $\mathcal{M}$ and hesitant fuzzy left ideal $\mathcal{L}$ on $S$ and $\mathcal{R}_{u}, \mathcal{M}_{u}, \mathcal{L}_{u} \subseteq$ $\varepsilon$ for all $u \in S$;

(3) $<\bar{r}_{\alpha}>_{R} \hat{\diamond}<\bar{m}_{\alpha}>_{M} \hat{\diamond}<\bar{l}_{\alpha}>_{L}=<\bar{r}_{\alpha}>_{R} \sqcap<\bar{m}_{\alpha}>_{M} \sqcap<\bar{l}_{\alpha}>_{L}$ for all $r, m, l \in S$ and $\alpha \subseteq \varepsilon$ such that $\alpha \neq \emptyset$;

(4) $<\bar{h}_{\alpha}>_{R} \hat{\diamond}<\bar{h}_{\alpha}>_{M} \hat{\diamond}<\bar{h}_{\alpha}>_{L}=<\bar{h}_{\alpha}>_{R} \sqcap<\bar{h}_{\alpha}>_{M} \sqcap<\bar{h}_{\alpha}>_{L}$ for all $h \in S$ and $\alpha \subseteq \varepsilon$ such that $\alpha \neq \emptyset$.

Proof. (1) $\Rightarrow(2)$.

Let $S$ be a regular ternary semigroup and let $\mathcal{R}, \mathcal{M}, \mathcal{L}$ be hesitant fuzzy right ideal, hesitant fuzzy lateral ideal and hesitant fuzzy left ideal on $S$ respectively. Now

$$
\begin{aligned}
& \mathcal{R} \hat{\diamond} \mathcal{M} \hat{\diamond} \mathcal{L} \sqsubseteq \mathcal{R} \hat{\diamond}\left[\chi_{S}^{(\varepsilon, \delta)}\right] \hat{\diamond}\left[\chi_{S}^{(\varepsilon, \delta)}\right] \sqsubseteq \mathcal{R}, \\
& \mathcal{R} \hat{\diamond} \mathcal{M} \hat{\diamond} \mathcal{L} \sqsubseteq\left[\chi_{S}^{(\varepsilon, \delta)}\right] \hat{\diamond} \mathcal{M} \hat{\diamond}\left[\chi_{S}^{(\varepsilon, \delta)}\right] \sqsubseteq \mathcal{M},
\end{aligned}
$$

$\mathcal{R} \hat{\diamond} \mathcal{M} \hat{\diamond} \mathcal{L} \sqsubseteq\left[\chi_{S}^{(\varepsilon, \delta)}\right] \hat{\diamond}\left[\chi_{S}^{(\varepsilon, \delta)}\right] \hat{\diamond} \mathcal{L} \sqsubseteq \mathcal{L}$. It implies $\mathcal{R} \hat{\diamond} \mathcal{M} \hat{\diamond} \mathcal{L} \sqsubseteq \mathcal{R} \sqcap \mathcal{M} \sqcap \mathcal{L}$.

As $S$ is regular, hence for any $x \in S$, there exist $h \in S$ such that $x=x h x$ and so $x=x h x=x h x h x$. Now

$$
\begin{aligned}
(\mathcal{R} \hat{\diamond} \mathcal{M} \hat{\diamond} \mathcal{L})_{x} & =\bigcup_{x=u v w}\left\{(\mathcal{R})_{u} \cap(\mathcal{M})_{v} \cap(\mathcal{L})_{w}\right\} \\
& \supseteq(\mathcal{R})_{x} \cap(\mathcal{M})_{h x h} \cap(\mathcal{L})_{x} \\
& \supseteq(\mathcal{R})_{x} \cap(\mathcal{M})_{x} \cap(\mathcal{L})_{x} \\
& =(\mathcal{R} \cap \mathcal{M} \cap \mathcal{L})_{x} .
\end{aligned}
$$

Therefore $\mathcal{R} \sqcap \mathcal{M} \sqcap \mathcal{L} \sqsubseteq \mathcal{R} \hat{\diamond} \mathcal{M} \hat{\diamond} \mathcal{L}$. Hence $\mathcal{R} \hat{\diamond} \mathcal{M} \hat{\diamond} \mathcal{L}=\mathcal{R} \sqcap \mathcal{M} \sqcap \mathcal{L}$.

It is easy to verify (2) $\Rightarrow(3)$ and (3) $\Rightarrow(4)$.

$(4) \Rightarrow(1)$. 
Let $h \in S$. Then $\bar{h}_{\alpha} \in<\bar{h}_{\alpha}>_{R} \sqcap<\bar{h}_{\alpha}>_{M} \sqcap<\bar{h}_{\alpha}>_{L}$. Given that $<\bar{h}_{\alpha}>_{R} \hat{\diamond}<\bar{h}_{\alpha}>_{M} \hat{\diamond}<\bar{h}_{\alpha}>_{L}=<\bar{h}_{\alpha}>_{R} \sqcap<\bar{h}_{\alpha}>_{M} \sqcap<\bar{h}_{\alpha}>_{L}$. Now

$$
\begin{aligned}
& \bar{h}_{\alpha} \in<\bar{h}_{\alpha}>_{R} \sqcap<\bar{h}_{\alpha}>_{M} \sqcap<\bar{h}_{\alpha}>_{L} \\
& =<\bar{h}_{\alpha}>_{R} \hat{\diamond}<\bar{h}_{\alpha}>_{M} \hat{\diamond}<\bar{h}_{\alpha}>_{L} \\
& \sqsubseteq \bar{h}_{\alpha} \hat{\diamond}\left[\chi_{S}^{(\varepsilon, \delta)}\right] \hat{\diamond} \bar{h}_{\alpha} \\
& \left.=\bigcup_{\bar{z}_{\gamma} \in\left[\chi_{S}^{(\varepsilon, \delta)}\right]}\left(\bar{h}_{\alpha} \hat{\diamond} \bar{z}_{\gamma} \hat{\diamond} \bar{h}_{\alpha}\right) \quad \text { (By the proposition } 20\right) \text {. }
\end{aligned}
$$

Hence from the above expression there exists $\bar{z}_{\alpha} \in H F P(S)$ such that $\bar{h}_{\alpha} \sqsubseteq$ $\bar{h}_{\alpha} \hat{\diamond} \bar{z}_{\alpha} \hat{\diamond} \bar{h}_{\alpha}=(\overline{h z h})_{\alpha}$. Thus by Proposition 20, $h=h z h$ for some $z \in S$. Hence $S$ is regular.

Definition 27. Let $S$ be a ternary semigroup and $\mathcal{H} \in H F(S)$. $\mathcal{H}$ is said to be idempotent if $\mathcal{H}=\mathcal{H} \hat{\diamond} \mathcal{H} \hat{\diamond} \mathcal{H}$.

Lemma 28. Let $S$ be a regular ternary semigroup. Then every hesitant fuzzy lateral ideal on $S$ is idempotent.

Proof. The proof is a straightforward.

Definition 29. A ternary semigroup $S$ is called left (resp. lateral, right) simple if $S$ contains no proper left (resp. lateral, right) ideal of $S$.

Definition 30. A ternary semigroup $S$ is called hesitant fuzzy left (resp. lateral, right) simple if every hesitant fuzzy left (resp. lateral, right) ideal on $S$ is constant function.

Theorem 31. Let $S$ be a ternary semigroup. Then $S$ is left (resp. lateral, right) simple if and only if $S$ is hesitant fuzzy left (resp. lateral, right) simple ternary semigroup.

Proof. Suppose $S$ is left simple and $\mathcal{H}$ is a hesitant fuzzy left ideal on $S$. Let $h, k \in S$. Then $S S h$ and $S S k$ are the left ideal of $S$. As $S$ is left simple, it implies $S S h=S$ and $S S k=S$. So there exists $t, u, v, w \in S$ s.t. $k=t u h$ and $h=v w k$. It follows that $\mathcal{H}_{k}=\mathcal{H}_{t u h} \supseteq \mathcal{H}_{h}=\mathcal{H}_{v w k} \supseteq \mathcal{H}_{k}$. Hence $\mathcal{H}_{h}=$ $\mathcal{H}_{k}$ for all $h, k \in S$. Therefore $\mathcal{H}$ is a constant function and hence $S$ is hesitant fuzzy left simple.

Conversely, suppose $S$ is hesitant fuzzy left simple and $A$ is a left ideal of 
$S$. By Lemma $8,\left[\chi_{A}^{(\varepsilon, \delta)}\right]$ is a hesitant fuzzy left ideal on $S$. Now by assumption, $\left[\chi_{A}^{(\varepsilon, \delta)}\right]$ is a constant function. As $A$ is a left ideal, it is non-empty. If $a \in A$, then $\left[\chi_{A}^{(\varepsilon, \delta)}\right]_{a}=\varepsilon$. Therefore for any element $h \in S,\left[\chi_{A}^{(\varepsilon, \delta)}\right]_{h}=\varepsilon$, which implies $h \in A$. Hence $S \subseteq A$. Consequently $S$ is simple.

\section{References}

[1] B. Davvaz, A. Zeb and A. Khan, On $(\alpha, \beta)$-fuzzy ideals of ternary semigroups, J. Indones. Math. Soc., 19, No 2 (2013), 123138.

[2] Y.B. Jun, K.J. Lee and S.Z. Song, Hesitant fuzzy bi-ideals in semigroups, Commun. Korean Math. Soc., 30, No 3 (2015), 143154.

[3] S. Kar and P. Sarkar, Fuzzy ideals of ternary semigroups, Fuzzy Inf. Eng., 2 (2012), 181-193.

[4] E. Kasner, An extension of the group concept, Bull. Amer. Math. Soc., 10 (1904), 290-291.

[5] D.H. Lehmer, A ternary analogue of abelian groups, AMS J. Math., 59 (1932), 329-338.

[6] M.Y. Abbasi, A.F. Talee, X.Y. Xie and S.A. Khan, Hesitant fuzzy ideal extension in po-semigroups, TWMS J. App. Eng. Math., (2017), Accepted.

[7] F.M. Sioson, Ideal theory in ternary semigroups, Math. Japan., 10 (1965), 63-84.

[8] V. Torra and Y. Narukawa, On HFSs and decision, in: The 18th IEEE International Conference on Fuzzy Systems, Jeju Island, Korea (2009), 13781382.

[9] V. Torra, Hesitant fuzzy sets, Int. J. Intell. Syst., 25 (2010), 529539.

[10] X.Y. Xie and J. Tang, Fuzzy radicals and prime fuzzy ideals of ordered semigroups, Information Sciences, 178 (2008), 43574374.

[11] L.A. Zadeh, Fuzzy sets, Inform. Control., 8 (1965), 338-353. 
